\title{
A note on frame transformations with applications to geodetic datums
}

\author{
Tomás Soler · John Marshall
}

\section{GPS Solutions (2003) 7:23-32}

The right expression for Eq. (10) is as follows:

$$
\begin{aligned}
\left\{v_{x}\right\}_{\text {ITRFyy }}= & \left\{\dot{T}_{x}\right\}+\llbracket\left(1+s\left(t_{k}\right)\right)[\dot{\varepsilon}]^{t}+\dot{s}[\delta \Re] \rrbracket\{x(t)\}_{\text {ITRF00 }}+\llbracket\left(1+s\left(t_{k}\right)\right)[\delta \Re]+\left(2 t_{D}-\left(t_{k}+t\right)\right) \\
& \times\left[\left(1+s\left(t_{k}\right)\right)[\dot{\dot{\varepsilon}}]^{t}+\dot{\boldsymbol{s}}[\delta \Re]\right] \rrbracket\left\{v_{x}\right\}_{\text {ITRF } 00}+\dot{s} \underline{[\dot{\varepsilon}]}^{t}\left\{2\left(t_{D}-t_{k}\right)\{x(t)\}_{\text {ITRF } 00}\right. \\
& \left.+\left(3 t_{D}^{2}+t_{k}^{2}-4 t_{D} t_{k}+2 t\left(t_{k}-t_{D}\right)\right)\left\{v_{x}\right\}_{\text {ITRF } 00}\right\}
\end{aligned}
$$

The previously published Table 2 was not properly aligned and contained some errors. Replace Table 2 by the following table:

\section{Table 2}

Transformation parameters and

\begin{tabular}{|c|c|c|c|c|c|}
\hline ITRF00 $\rightarrow$ & & $\begin{array}{l}\text { NAD83 } \\
t_{k}=1997.0\end{array}$ & $\begin{array}{l}\text { ITRF89 } \\
t_{k}=1988.0\end{array}$ & $\begin{array}{l}\text { ITRF92 } \\
t_{k}=1988.0\end{array}$ & $\begin{array}{l}\text { ITRF94 } \\
t_{k}=1997.0\end{array}$ \\
\hline$T_{x}$ & $\mathrm{~cm}$ & 99.56 & 2.97 & 1.47 & 0.67 \\
\hline$T_{y}$ & $\mathrm{~cm}$ & -190.13 & 4.75 & 1.35 & 0.61 \\
\hline$T_{z}$ & $\mathrm{~cm}$ & -52.15 & -7.39 & -1.39 & -1.85 \\
\hline$\varepsilon_{x}$ & mas & 25.915 & 0.00 & 0.00 & 0.00 \\
\hline$\varepsilon_{y}$ & mas & 9.426 & 0.00 & 0.00 & 0.00 \\
\hline$\varepsilon_{z}$ & mas & 11.599 & 0.18 & 0.18 & 0.00 \\
\hline$s$ & $\mathrm{ppb}$ & 0.62 & 5.85 & 0.75 & 1.55 \\
\hline$\dot{T}_{x}$ & $\mathrm{~cm} / \mathrm{y}$ & 0.07 & 0.00 & 0.00 & 0.00 \\
\hline$\dot{T}_{y}$ & $\mathrm{~cm} / \mathrm{y}$ & -0.07 & -0.06 & -0.06 & -0.06 \\
\hline$\ddot{T}_{z}$ & $\mathrm{~cm} / \mathrm{y}$ & 0.05 & -0.14 & -0.14 & -0.14 \\
\hline$\dot{\varepsilon}_{x}$ & mas/y & 0.013 & 0.00 & 0.00 & 0.00 \\
\hline$\dot{\varepsilon}_{y}$ & mas/y & -0.015 & 0.00 & 0.00 & 0.00 \\
\hline$\dot{\varepsilon}_{z}$ & mas/y & -0.020 & -0.02 & -0.02 & -0.02 \\
\hline$\dot{s}$ & $\mathrm{ppb} / \mathrm{y}$ & -0.18 & 0.01 & 0.01 & 0.01 \\
\hline
\end{tabular}
their rates from ITRF00 to other frames
Note: All rotations are given counter-clockwise positive; mas $=$ milliarc second; $\mathrm{ppb}=$ parts per billion $=$ $10^{-6} \mathrm{ppm}$

The online version of the original article can be found at http:// dx.doi.org/10.1007/s10291-003-0044-8

Published online: 25 July 2003

(C) Springer-Verlag 2003

T. Soler $(\varangle) \cdot$ J. Marshall

National Geodetic Survey, NOS NOAA,

N/NGS22 \#8825, 1315 East-West Highway,

Silver Spring, MD 20910-3282, USA

Tel.: +1-301-7133205

Fax: +1-301-7134324

E-mail: Tom.Soler@noaa.gov 
The following two equations should be replaced in Appendix B.

$$
\begin{aligned}
\partial \mathscr{A} / \partial\left\{\varepsilon\left(t_{k}\right)\right\}= & \partial[\partial \varepsilon] / \partial t_{D} \\
= & \left(1+s\left(t_{k}\right)\right) \underline{\left[v_{x}\right]} \\
& +\dot{s} \llbracket\left[\underline{\llbracket x]}+\left(2 t_{D}-\left(t_{k}+t\right)\right) \underline{\left[v_{x}\right] \rrbracket}\right. \\
= & {[\bar{\partial} \varepsilon] } \\
\partial \mathscr{A} / \partial\{\dot{\varepsilon}\}= & \partial[\partial \dot{\varepsilon}] / \partial t_{D} \\
= & \left(1+s\left(t_{k}\right)\right) \underline{\llbracket[x]}+\left(2 t_{D}-\left(t_{k}-t\right)\right) \underline{\left[v_{x}\right] \rrbracket} \\
+ & \dot{s} \llbracket 2\left(t_{D}-t_{k}\right) \underline{[x]}+\left(3 t_{D}^{2}+t_{k}^{2}-4 t_{D} t_{k}\right. \\
+ & \left.2 t\left(t_{k}-t_{D}\right)\right) \underline{\left[v_{x}\right] \rrbracket}=[\bar{\partial} \dot{\varepsilon}]
\end{aligned}
$$

